\title{
Surgical Outcomes of Combined Phacoemulsification and Silicone oil Removal in Post Retinal Detachment Surgery Patients
}

\author{
Uzma Haseeb ${ }^{1}$, Muhammad Haseeb ${ }^{2}$, Aziz-ur-Rehman ${ }^{3}$ \\ ${ }^{1-3}$ Department of Ophthalmology, Al Ibrahim Eye Hospital, Karachi, ${ }^{2}$ Al-Ain Institute of Eye Diseases, Karachi
}

\begin{abstract}
Purpose: To evaluate the surgical outcomes of combined Phacoemulsification with IOL implantation and Silicone oil removal in patients with previous retinal detachment surgery.
\end{abstract}

Study Design: Interventional case series.

Place and Duration of Study: Al-lbrahim eye Hospital Karachi from January 2019 to December 2019.

Methods: Patients who had previous retinal detachment surgery with silicone oil used as an internal tamponade with post-operative cataract formation and attached retina confirmed by indirect ophthalmoscopic examination or B scan depending on media clarity were included in the study. The time interval between the two surgeries was 8 to 10 months. Phacoemulsification with IOL implantation was done followed by silicone oil removal using 23gauge pars plana method. Post-operative follow-ups were done at day 1,1 month and at 6 months to record visual acuity and retinal status.

Results: Thirty eyes of 30 patients between ages of 25 to 65 years were included in the study Best corrected visual acuity was recorded pre and post-operatively using Snellen chart. Pre-operatively visual acuity was 6/60 in 12 patients, 6/95 in 12 patients and 6/120 in 6 patients. There was improvement of 2 lines of visual acuity post operatively as recorded by Snellen chart. Retina was attached in $28(93.33 \%)$ patients while $2(6.66 \%)$ patients had re-detached retina at the end of 6 months.

Conclusion: Combined Phacoemulsification with Silicone oil removal is a useful method that is associated with good visual and anatomical outcomes and it eliminates the need for third surgery for cataract extraction.

Key Words: Retinal Detachment, Phacoemulsification, Silicon Oil.

How to Cite this Article: Haseeb $U$, Haseeb $M$, Rehman A. Surgical Outcomes of Combined Phacoemulsification and Silicone Oil Removal in Post Retinal Detachment Surgery Patients. Pak J Ophthalmol. 2020; 36 (4): 423-427.

Doi: https://doi.org/10.36351/pjo.v36i4.1116

\section{INTRODUCTION}

Proliferative vitreoretinopathy (PVR) is one of the complications of rhegmatogeneous retinal detachment.

Correspondence: Uzma Haseeb

Department of Ophthalmology

Al-Ibrahim Eye Hospital, Karachi

Email:uzma_123us@yahoo.com

Received: August 5, 2020

Accepted: September 5, 2020
Silicone oil is used as an internal tamponade to reduce the rate of retinal re-detachment (RRD). In complicated cases of retinal detachment such as in giant retinal tears, trauma, proliferative vitreoretinopathy (PVR), and diabetic tractional retinal detachment silicone oil is used as an effective tamponade. Silicone oil gives better view for retinal examination while it is impossible if gas or air is used as tamponade in eye.

After silicone oil removal the incidence of retinal re-detachment (RRD) vary widely from $6 \%$ to $28 \%$. 
One of most important risk factor for retinal redetachment is previously unsuccessful surgery. ${ }^{1}$ Most common complication that can occur after cataract surgery including phacoemulsification is retinal detachment (RD). Although with each passing day new advances had been introduced in vitreoretinal surgery but still RD can cause severe visual loss and thus leads to blindness. After phacoemulsification the risk of RD is $0.32 \%$ to $1.17 \%$. $^{2}$ In general population the incidence of RRD is between $0.01 \%$ and $0.02 \%$ and is more common in males. Around the sixth decade of life the incidence of RRD is at its peak and right eye is more affected then left eye. ${ }^{3}$

Risk of RRD differs with ethnicity, it is tenfold more in Caucasians than African populations. ${ }^{4}$ In Asian population the incidence is variable but more in young age group. East Asians are at same risk as Caucasians ${ }^{5}$ but risk of RRD is lower in South Asians, which is threefold less than Caucasians. Common risk factors associated with RRD are Myopia, fellow eye RRD, trauma and proliferative diabetic retinopathy. Previously ND: YAG laser capsulotomy was considered as one of risk factor for RRD but this has been questioned recently and is under debate. ${ }^{6}$ Posterior vitreous detachment (PVD) associated with retinal tear or break can lead to RD in $80-90 \%$ of cases. Around 60 to 70 years of age there is increased liquefaction of vitreous gel, which is natural and age related process and can lead to PVD. At the time of cataract surgery complete PVD without RD is considered beneficial because it gives protection against RD. ${ }^{7}$ In posterior segment surgery Silicone Oil (SO) is used as an endo tamponade to flatten retina and to maintain intraocular pressure (IOP). However, silicone oil emulsification, secondary glaucoma, cataract, and corneal degeneration are vision threatening complications of SO. That is why it is preferred to remove SO from eye after its tamponading effects are no more required. Silicone oil when comes in contact with lens leads to formation of posterior sub capsular cataracts, mostly $6-12$ months after surgery. After development of secondary cataract in SO filled eye combination surgery including cataract extraction with removal of SO is advisable. ${ }^{8}$

This study was designed to evaluate the rate of Retinal re-detachment and visual outcomes after combined phacoemulsification and IOL implantation and silicone oil removal in patients who had previously undergone retinal detachment surgery.

\section{METHODS}

This was an interventional case series with nonprobability convenience sampling technique. Study was carried out at Al-Ibrahim Eye Hospital, Karachi from January 2019 to December 2019. A prior ethical approval was taken from the institutional review board of Isra postgraduate institute of Ophthalmology, AlIbrahim Eye Hospital, Karachi. Informed written consent was taken from all the study participants. Patients who had previous retinal detachment surgery with silicone oil used as an internal tamponade with post-operative cataract formation and attached retina confirmed by indirect ophthalmoscopic examination or B scan depending on media clarity were included in the study. Indications of retinal detachment surgery with use of SO in these patients were rhegmatogeneous retinal detachment (RD) 13 (43.33\%) patients, Tractional RD 13 (43.33\%) patients and combined tractional + rhegmatogeneous RD 4 (13.33\%) patients. Patients' age was 25 years or above with no discrimination of gender. Exclusion criteria were retinal detachment surgery with tamponading agents other than silicone oil, other ocular pathology, aphakia, pseudophakic eyes, and Post traumatic eyes with retinal detachment.

A well trained vitreoretinal surgeon performed all the surgeries to reduce chances of bias. Phacoemulsification with IOL implantation was done first, followed by removal of silicone oil through $23 \mathrm{G}$ pars plana technique. Fluid-air exchange was done at least two times. Follow-up of all patients was planned at first day of surgery then first month, and sixth month after surgery.

At each follow-up best-corrected visual acuity, slit lamp $90 \mathrm{D}$ fundus examination, Intra ocular pressure and anatomical retinal attachment were assessed. If retina was completely attached at last follow-up (6 months), it was considered as anatomically attached retina. If ongoing (PVR) proliferative vitreoretinopathy or the contractions of the retina leading to RD within six months of SO removal was detected, it was considered as anatomically detached retina meaning failed surgery.

Statistical package for social sciences (SPSS) version 23.0 was used for statistical analysis. Quantitative variables like age, duration between two surgeries, and intraocular pressure were presented as mean $\pm \mathrm{SD}$. The data for gender, reason for previous surgery, tamponade, visual acuity (pre-operative and at 
6 month follow-up) and state of retina were described by frequency and percentages.

\section{RESULTS}

In this study total 30 patients are recruited. Mean age of patients was $52.35 \pm 9.8$ with minimum age of 25 and maximum age of 65 years. There were 20 $(66.66 \%)$ male and $10(33.33 \%)$ female patients. Visual acuity was recorded pre-operatively and postoperatively by Snellen chart. For details see table 1 .

Table 1:

\begin{tabular}{ccl}
\hline $\begin{array}{c}\text { Pre-operative } \\
\text { Visual Acuity }\end{array}$ & $\begin{array}{c}\text { No of } \\
\text { Patients }(\%)\end{array}$ & $\begin{array}{c}\text { Post op Visual Acuity } \\
\text { at 6 Month }\end{array}$ \\
\hline $6 / 60$ & $12(40 \%)$ & $\begin{array}{l}\text { All 12 Patients Improved } \\
\text { to VA }=6 / 38 \\
6 \text { Patients Improved } \\
\text { to VA }=6 / 75\end{array}$ \\
$6 / 95$ & $12(40 \%)$ & $\begin{array}{l}\text { 6 Patients Improved } \\
\text { to VA }=6 / 60 \\
\text { 3 Patients Improved } \\
\text { to VA }=6 / 75 \\
\text { 3 Patients Improved } \\
\text { to VA }=6 / 60\end{array}$ \\
$6 / 120$ & $6(20 \%)$ & \\
& &
\end{tabular}

Status of retina was also assessed at the end of 6 months. Twenty-eight $(93.33 \%)$ patients had attached retina while $2(6.66 \%)$ of them presented with retinal re-detachment (Graph 1).

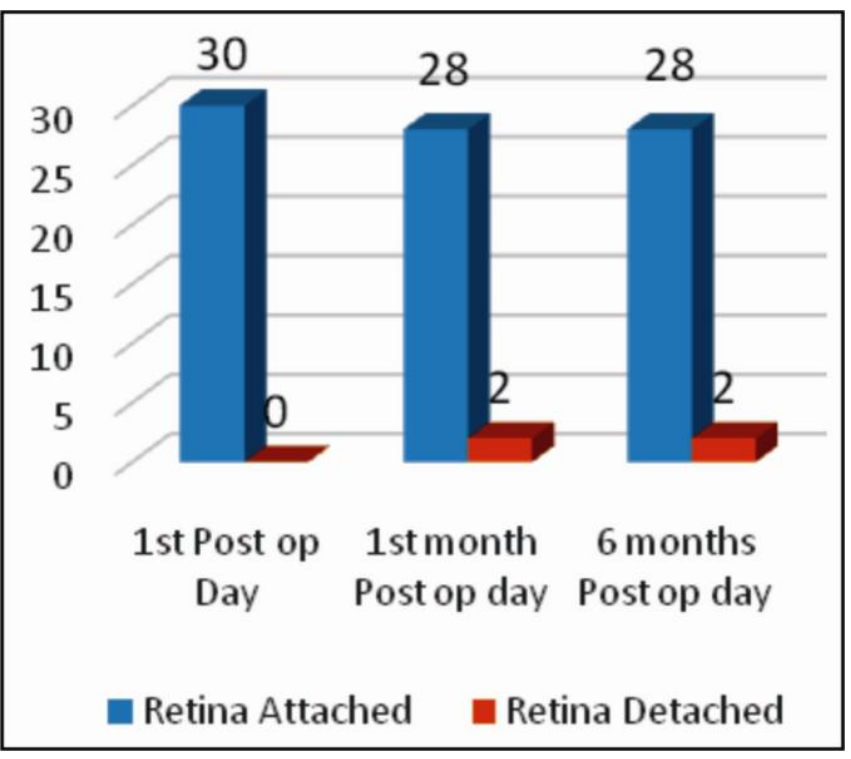

Fig. 1: Status of retinal at follow-up.

\section{DISCUSSION}

In complicated cases of RD three port pars plana vitrectomy with silicone oil as internal tamponade is extensively used to flatten retina. Most frequent long term ocular complications of SO are cataract, glaucoma and band keratopathy. ${ }^{9}$ The longer the duration of SO in the eye the higher will be the chances of development of cataract and glaucoma. ${ }^{10}$ In some cases, even very short duration of intraocular silicone oil tamponade can cause cataract formation. ${ }^{11}$ The mechanical effect of silicone oil in cataract formation is more than the toxic effect of silicone oil. ${ }^{12}$ It has been shown that mechanical energy from intraocular instruments causes emulsification of silicone oil. ${ }^{13}$ It seems reasonable to do combined procedure including cataract extraction with SO removal in one sitting. Measures should be adopted for safe removal of silicone as sometimes removal of silicone oil can lead to retinal re-detachment. Combining the two surgeries leads to shortened total surgical time. ${ }^{14}$

Visual outcomes are also good when two surgical procedures are done at the same time. On the other hand, this combined procedure is associated with some potential disadvantages like difficulty in intraocular lens power calculation. Silicone oil contact with corneal endothelium during surgery can lead to corneal damage and dislocation of intraocular lens in posterior segment of the eye can also occur if the posterior capsulorhexis is too large. There are several methods of this combined procedure in literature. These methods usually differ from each other in silicone oil removal technique. Some of these are removal of Silicone oil through a planned posterior capsulorhexis and removal of silicone oil through a trans-scleral approach. ${ }^{15.16,17}$

In the present study, visual acuity was documented pre and post-operatively which showed improvement in majority of patients. Antoun et al reported similar results in patients and showed better visual outcomes or better post-operative visual acuity. ${ }^{18}$ Ramezani et al stated that more patients had an increased or unchanged visual acuity (VA) at the last follow-up visit. Rate of retinal re-detachment was low at 6 month follow-up. ${ }^{19}$

In the current study, all patients had previous retinal detachment surgery with SO used as an internal tamponade. In comparison to our study, study done by Nagpal et al showed detachment to be $12.7 \% .^{20}$ 
Another study by Jain et al documented $11.6 \%$ redetachment rate. ${ }^{21}$ Furthermore, different studies by Mancino $\mathrm{R}$ et $\mathrm{al}^{22}$ reported $20.2 \%$ and Choudhary MM et $\mathrm{al}^{23}$ reported $27.6 \%$ re-detachment. Yu J et al reported that $81.3 \%$ of the patients after removal of silicone oil were observed to have retinal redetachment in first 10 days. ${ }^{24}$ The most common cause after SO removal of RRD in first 10 days was residual vitreoretinal tractions at the vitreous base. Caramoy et al stated that $\mathrm{SO}$ used as endotamponade after vitrectomy could lead to temporary increase in IOP and did not cause any damage on removal of SO. ${ }^{25}$

Limitation of this study is the small sample size and shorter duration of follow-up. Retention of SO in eye for long duration does not give any additional advantages to the eye. Although there is still a risk of retinal re-detachment after silicone oil removal so strict follow-up is the main element in these cases.

\section{CONCLUSION}

Visual outcomes were found to be good with low rate of retinal re-detachment after 6 month follow-up of combined phacoemulsification with IOL implantation followed by silicone oil removal. Combined phacoemulsification with silicone oil removal is a preferred method that is associated with better visual and anatomical outcomes. This also eliminates the chances of patients undergoing third surgery for cataract extraction.

\section{Ethical Approval}

The study was approved by the Institutional review board/Ethical review board.

\section{Conflict of Interest}

Authors declared no conflict of interest.

\section{REFERENCES}

1. Rhatigan M, McElnea E, Murtagh P, Stephenson K, Harris E, Connell P, et al. Final anatomic and visual outcomes appear independent of duration of silicone oil intraocular tamponade in complex retinal detachment surgery. Intern J Ophthalmol. 2018; 11 (1): 83.

2. Grzybowski A, Kanclerz P. Does Nd: YAG capsulotomy increase the risk of retinal detachment? Asia-Pac J Ophthalmol. 2018; 7 (5): 339-344.
3. Mitry DCD, Yorston D, Siddiqui MA, Campbell H, Murphy AL, Fleck BW, et al. Scottish RD Study Group. The epidemiology and socioeconomic associations of retinal detachment in Scotland: a twoyear prospective population-based study. Investig Ophthalmol Vis Sci. 2010; 51: 4963-4968.

4. Bechrakis NE, Dimmer A. Rhegmatogenous retinal detachment: epidemiology and risk factors. Der Ophthalmologe. 2018; 115 (2): 163-178.

5. Kirin M, Chandra A, Charteris DG, Hayward C, Campbell S, Celap I, et al. Genome-wide association study identifies genetic risk underlying primary rhegmatogenous retinal detachment. Hum Mol Genet. 2013; 22 (15): 3174-3185.

6. Liu X, Wang Q, Zhao J. Acute retinal detachment after Nd: YAG treatment for vitreous floaters and posterior capsule opacification: a case report. BMC Ophthalmology, 2020; 20 (1): 1-4.

7. Mahroo OA, Dybowski R, Wong R, Williamson TH. Characteristics of rhegmatogenous retinal detachment in pseudophakic and phakic eyes. Eye, 2012; 26 (8): 1114-1121.

8. Al-Wadani SF, Abouammoh MA, Abu El-Asrar AM. Visual and anatomical outcomes after silicone oil removal in patients with complex retinal detachment. Int Ophthalmol. 2014; 34 (3): 549-556.

9. Yang JY, Kim HK, Kim SH, Kim SS. Incidence and risk factors of cystoid macular edema after vitrectomy with silicone oil tamponade for retinal detachment. Korean Journal of Ophthalmology, 2018; 32 (3): 204 210.

10. Jiang Y, Oh DJ, Messenger W, Lim JI. Outcomes of 25 -gauge vitrectomy with relaxing retinectomy for retinal detachment secondary to proliferative vitreoretinopathy. Journal of Vitreoretinal Diseases, 2019; 3 (2): 69-75.

11. Dhalla K, Kapesa I, Odouard C. Incidence and risk factors associated with retinal redetachment after silicone oil removal in the African population. International Ophthalmology, 2017; 37 (3): 583-589.

12. Zhang X, Pan Y, Song Z. Trocar Opening: A Novel Management Strategy for Silicone Oil Removal with Phacoemulsification and IOL Implantation. J Clin Exp Ophthalmol. 2018; 9 (6): 1000772.

13. Francis JH, Latkany PA, Rosenthal JL. Mechanical energy from intraocular instruments causes emulsification of silicone oil. Br J Ophthal. 2017; 91: 818-821.

14. Feng X, Li C, Zheng Q, Qian X, Shao W, Li Y, et al. Risk of silicone oil as vitreous tamponade in pars plana vitrectomy: a systematic review and meta-analysis. Retina, 2017; 37 (11): 1989-2000. 
15. Xu W, Cheng W, Zhuang H, Guo J, Xu G. Safety and efficacy of transpupillary silicone oil removal in combination with micro-incision phacoemulsification cataract surgery: comparison with 23-gauge approach. BMC Ophthalmology, 2018; 18 (1): 200.

16. Zhou C, Qiu Q. $360^{\circ}$ versus localized demarcation laser photocoagulation for macular-sparing retinal detachment in silicone oil-filled eyes with undetected breaks: A retrospective, comparative, interventional study. Lasers Surg Med. 2015; 47 (10): 792-797.

17. Romano MR, Vinciguerra R, Vinciguerra $P$. Sutureless silicone oil removal: a quick and safe technique. Retina, 2013; 33 (5): 1090-1091.

18. Antoun J, Azar G, Jabbour E, Kourie HR, Slim E, Schakal A, et al. Vitreoretinal surgery with silicone oil tamponade in primary uncomplicated rhegmatogenous retinal detachment. Retina, 2016; 36 (10): 1906-1912.

19. Ramezani A, Ahmadieh H, Rozegar A, Soheilian M, Entezari M, Moradian S, et al. Predictors and outcomes of vitrectomy and silicone oil injection in advanced diabetic retinopathy. Korean J Ophthalmol. 2017; 31 (3): 217-229.

20. Nagpal MP, Videkar RP, Nagpal KM. Factors having implications on retinal detachments after silicone oil removal. Indian J Ophthalmol. 2012; 60 (6): 517-520.

21. Jain N, McCuen BW, Mruthyunjaya P. Unanticipated vision loss after pars plana vitrectomy. Surv Ophthalmol. 2012; 57 (2): 91-104.

22. Mancino R, Aiello F, Ciuffoletti E, Di Carlo E, Cerulli A, Nucci C. Inferior retinotomy and silicone oil tamponade for recurrent inferior retinal detachment and grade C PVR in eyes previously treated with pars plana vitrectomy or scleral buckle. BMC Ophthalmology, 2015; 15 (1): 173.
23. Choudhary MM, Choudhary MM, Saeed MU, Ali A. Removal of silicone oil: prognostic factors and incidence of retinal re-detachment. Retina, 2012; 32 (10): 2034-2038.

24. Yu J, Zong Y, Jiang C, Zhu H, Deng G, Xu G. Silicone Oil Emulsification after Vitrectomy for Rhegmatogenous Retinal Detachment. J Ophthalmol. 2020; 2020: 1-6.

25. Caramoy A, Kearns VR, Chan YK. Development of emulsification resistant heavier-than-water tamponades using high molecular weight silicone oil polymers. J Biomater Appl. 2015; 30 (2): 212-220.

\section{Authors' Designation and Contribution \\ Uzma Haseeb; Assistant Professor: Concepts, Design, Literature Research, Data Acquisition, Data Analysis, Manuscript Preparation, Manuscript Editing. \\ Muhammad Haseeb; Consultant Ophthalmologist: Design, Literature Research, Manuscript Preparation.}

Aziz-ur-Rehman; Professor: Manuscript Preparation, Manuscript Editing, Manuscript Review. 